\title{
Determination of Bifurcation Angles of the Retinal Vascular Tree through Multiple Orientation Estimation based on Regularized Morphological Openings
}

\author{
Sandra Morales ${ }^{1,2}$, Álvar-Ginés Legaz-Aparicio ${ }^{3}$, Valery Naranjo ${ }^{1,2}$ and Rafael Verdú-Monedero ${ }^{3}$ \\ ${ }^{1}$ Instituto Interuniversitario de Investigación en Bioingeniería y Tecnología Orientada al Ser Humano, Universitat \\ Politècnica de València, I3BH/LabHuman, Camino de Vera s/n, 46022 Valencia, Spain \\ ${ }^{2}$ Grupo Tecnologías de Informática Aplicadas a la Oftalmología, Unidad Conjunta UPV- FISABIO, Valencia, Spain \\ ${ }^{3}$ Universidad Politécnica de Cartagena, Cartagena, Spain
}

Keywords: Retinal Image, Bifurcation Angle, Multiple Orientation Estimation, Regularized Morphological Opening.

Abstract: $\quad$ This paper describes a new approach to compute bifurcation angles in retinal images. This approach is based on the estimation of multiple orientations at each pixel of a gray retinal image. The main orientations are provided by directional openings whose outputs are regularized in order to extend the orientation information to the whole image. The detection of vessel bifurcations is based on the coexistence of two or more than two different main orientations at the same pixel. Once the bifurcations and crossovers has been identified, bifurcation angles are calculated. The proposed procedure of computing bifurcation angles by means of orientation estimation at all pixels of the gray level image is much more stable than those methods which are based on the skeleton of the vascular tree, since a slight variation of a pixel of the skeleton can produce a significant change in the angle value.

\section{INTRODUCTION}

Retinal vasculature is able to indicate the status of other vessels of the human body. Its study involves a non-invasive or minimally invasive procedure and, usually, it is included in the standard screening of any patient with diseases in which the vessels may be altered. Specifically, the identification and study of bifurcations and crossovers has great significance in cardiovascular diseases as well as in their early detection (Azzopardi and Petkov, 2011). Moreover, vascular changes produced in systemic diseases usually induce particular modifications in the vessels, such as changes in bifurcation angles. Based on this fact, this paper is focused on automatically determining the bifurcation angles calculated on vessel bifurcations previously detected. There are two approaches to detect vessel bifurcations: methods based on geometrical features and methods based on models (Abramoff et al., 2010). The method proposed in this paper belongs to the first group. In particular, the detection of vessel bifurcations is addressed by analyzing a multiple orientation vector field provided by the regularization of directional morphological openings. The orientation field is given by a directional signature
(Soille and Talbot, 2001) for each pixel using a set of directional openings with a line segment. Then, the orientation of a pixel is defined as the one associated to the directional opening which produces the maximum value of the signature of this pixel. Nevertheless, the original approach from (Soille and Talbot, 2001) does not deal with the multiple orientation case and does not take into account that, locally, pixels in natural images can be associated to more than one orientation, e.g., crossing lines, corners and junctions (also known as X-, L- and Y-junctions, respectively). To determine not only the main direction but all the significant orientations, the directional signature is analyzed in the present work using multiple peak detection on the curve interpolated by b-splines. Thanks to the estimation of the main orientations of each bifurcation point of the retinal images, the angles generated by the vessels on all of them can be analyzed.

In the literature, the most common approach to detect retinal tree bifurcations and their corresponding bifurcation angles is through the analysis of the segmented vascular tree. In general, this segmentation can be performed by matched filters (Chaudhuri et al., 1989), by region growing and scale-space anal-

126 Morales S., Legaz-Aparicio Á., Naranjo V. and Verdú-Monedero R. 
ysis (Martínez-Pérez et al., 1999), by mathematical morphology (Zana and Klein, 2001), etc. Regarding the detection of vessel bifurcations and crossovers on segmented retinal images it can be done, e.g., by using a set of trainable keypoint detectors and a bank of Gabor filters (Azzopardi and Petkov, 2013), or with matched filters (Ardizzone et al., 2008). The measurement of the bifurcation angles is usually carried out fitting the segmented vessels, or their skeleton, by straight lines and then estimating the angle between them (Gao et al., 2000; Martinez Perez et al., 2002; MacGillivray et al., 2012). Segmentation-dependent results are the major drawback of this approach: a slight variation of a pixel in the skeleton results can produce a significant change in the angle value, even of several degrees.

This paper is organized as follows: Section 2 firstly addresses the method to estimate multiple orientations at each pixel, and then the approach followed to detect the bifurcations and crossovers in retinal images together with the calculation of the bifurcation angles is described. Section 3 shows the results of the proposed method in a public database. Finally, Section 4 closes the paper with the conclusions and future work lines.

\section{METHOD}

The goal of this paper is to propose a new method to compute bifurcation angles of the retinal tree from a gray image without resorting to the skeletonization of a segmented (binary) image. For that purpose, three stages are necessary: estimation of the multiple orientation vector field, detection of bifurcations and crossovers and, finally, the measurement of the desired angles. The key of the method is the way to estimate the multiple orientations existing in a fundus image. The algorithm proposed to carried out this estimation is depicted in Figure 1. This approach differs from (Angulo et al., 2011), where the multiple main orientations are estimated by analysing a block of the image, whereas in this paper the multiple orientations are estimated at each pixel.

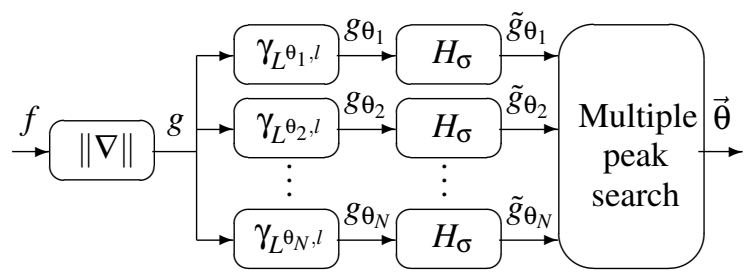

Figure 1: Flowchart of the multiple orientation estimation method.

\subsection{Multiple Orientation Estimation}

Let $f(\mathbf{x}): E \rightarrow \mathbb{R}$ be a gray-level image, where the support space is $E \subset \mathbb{Z}^{2}$ and the pixel coordinates are $\mathbf{x}=(x, y)$. Let us define $g(\mathbf{x})$ as the absolute value of the gradient of $f(\mathbf{x})$, i.e.,

$$
g(\mathbf{x})=\|\nabla f(\mathbf{x})\|=\sqrt{\left(\frac{\partial f(x, y)}{\partial x}\right)^{2}+\left(\frac{\partial f(x, y)}{\partial y}\right)^{2}} .
$$

The directional opening of $g(\mathbf{x})$ by a linear (symmetric) structuring element (SE) of length $l$ and direction $\theta$ is defined as the directional erosion of $g$ by $L^{\theta, l}$ followed by the directional dilation with the same SE (Verdú-Monedero et al., 2011):

$$
\gamma_{L^{\theta, l}}(g)(\mathbf{x})=\delta_{L^{\theta, l}}\left[\varepsilon_{L^{\theta, l}}(g)\right](\mathbf{x}),
$$

where the directional erosion and dilation are respectively

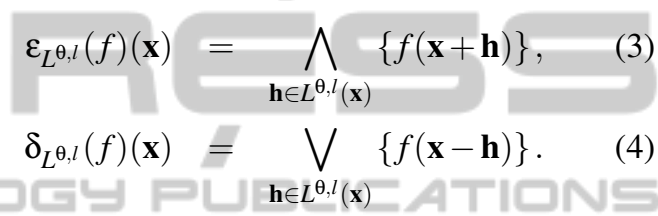

The proposed orientation model is based on a decomposition of the gradient information by families of linear openings, $\left\{\gamma_{L^{\theta_{i}}, l}\right\}_{i \in I}$, according to a particular discretization of the orientation space $\left\{\theta_{i}\right\}_{i \in I}$.

In the next step of the proposed method, a filtering is performed at each one of the directional openings (depicted as $H_{\sigma}$ in Figure 1). The filtering diffuses the orientation information and avoids angle mismatches due to noise. The kernel $H_{\sigma}$ is the sampling of the gaussian low-pass filter

$$
H_{\sigma}\left(\omega_{1}, \omega_{2}\right)=e^{-\frac{\sigma^{2}\left(\omega_{1}^{2}+\omega_{2}^{2}\right)}{2}},
$$

where $\sigma$ is the spatial standard deviation of the filter. More details of the method can be found in (LegazAparicio et al., 2014).

Once the directional openings have been filtered, the directional signature at pixel $\mathbf{x}$ is defined as

$$
s_{\mathbf{x} ; l}(i)=\tilde{g}_{\theta_{i}}(\mathbf{x}) .
$$

Then, $s_{\mathbf{x} ; l}(i)$ is interpolated using cubic b-splines and its maxima correspond to the multiple orientations existing at pixel $\mathbf{x}$. Finally, collecting all the orientations estimated at all the pixels in the image provides the multidimensional vector field $\vec{\theta}(\mathbf{x})$.

\subsection{Detection of Bifurcations and Crossovers}

The method previously presented can be applied to a gray or on a binary image. The resulting orientation vector field can be used then to detect the existing bifurcations and crossovers of the retinal tree 
(see Figure 2). In general, most of the methods which detect significant points of a vascular tree are based on a previously segmented, and therefore binary, image. In this paper, the segmentation stage is skipped and the method is applied directly to a gray image, avoiding the segmentation result dependence. However, the method can also be applied to a segmented image, providing a better orientation field due to the binarization of the image.
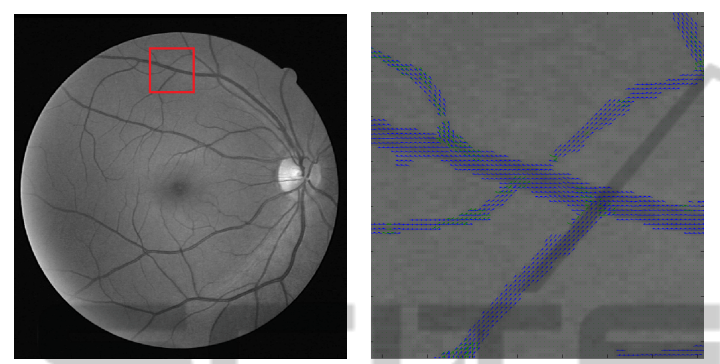

Figure 2: Left: real retinal image. Right: close-up of the image with its estimated multiple orientation vector field.

The detection procedure takes into account only the two main orientations at each pixel. The method considers that a bifurcation or crossover exists if two conditions happen. The first condition is that the magnitude of the regularized opening has to be greater than a threshold, $\left|\tilde{g}_{\theta_{i}}\right|>t h$. The second condition to be fulfilled is that the two main orientations must differ more than $2 \Delta \theta$, where $\Delta \theta$ is the angular resolution of the multiple orientation estimation method. After processing these two conditions, a refinement stage is necessary to reduce false positives and then to discriminate between bifurcations or crossings and high curvature areas.

\subsection{Classification of Detected Points}

In order to determine which interest points are bifurcations and crossovers and which ones are high curvature areas, a control area around each point of interest is established. The control area is a window with circular shape centred in the point of interest. The radius of the window must be large enough to contain the vessels belonging to the point of interest. At this stage, a coarse segmentation based on a top-hat morphological filter followed by a threshold is performed. The aim of this segmentation is to speed up the classification stage.

The classification is carried out by placing a circular window at the interest point and analyzing the number of branches that cross the circle defined by the mask. According to the number of branches that cross the circle, the points of interest are classified

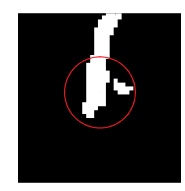

(a)

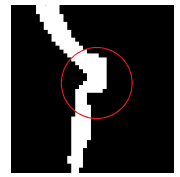

(b)

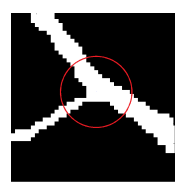

(c)

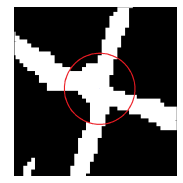

(d)
Figure 3: Circular windows placed at different detected interest points with two main orientations.

into four types:

- If there is only one branch, the point of interest is a vessel termination (see Figure 3(a)).

- If there are two branches, the point of interest is a vessel with high curvature (see Figure 3(b)).

- If there are three branches (in general, an odd number greater than one), the point of interest will be a bifurcation (see Figure 3(c)).

- Finally, if there are four branches (in general, an even number greater than two), the point of interest will be a crossover (see Figure 3(d)).

Since we are interested in detecting bifurcations and crossovers, only the points of interest whose mask is crossed by more than two branches will be considered. Figure 4(a)-(b) show the detected interest points and Figure 4(c)-(d) show the bifurcations and crossover after the classification stage.

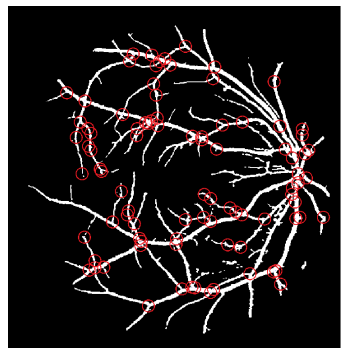

(a)

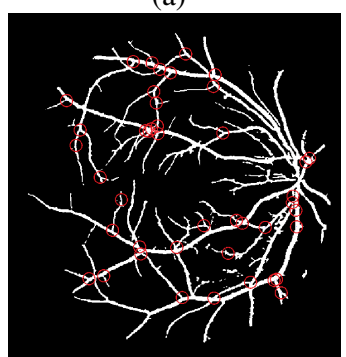

(c)

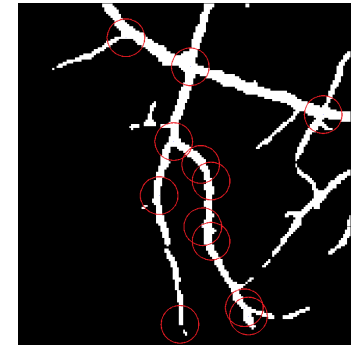

(b)

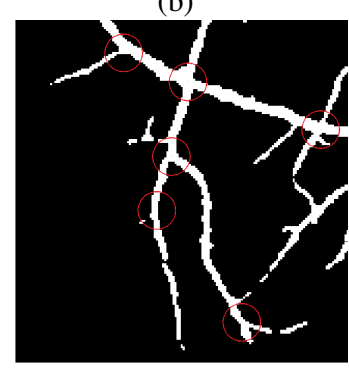

(d)
Figure 4: (a) Detection of interest points: bifurcations, crossings and high curvature areas. (b) Close-up of (a). (c) Bifurcations after the classification. (d) Close-up of (c). 


\subsection{Measurement of Bifurcation Angles}

Once the bifurcations have been classified, it is possible to measure the bifurcation angles. The angle is calculated by performing the difference between the orientation vectors of each branch. From the intersection of the circular window and the branch, an interior point is chosen as representative of this branch, and its orientation vector will define the orientation of the branch at this pixel. Note that the choice of this pixel is not critical since the orientation vector field varies slowly inside the vessel. Due to the discretization of the orientation space of the multiple orientation method, the orientation is estimated using the ASGVF method (Verdú-Monedero et al., 2011) which achieves a higher angular resolution. Since the ASGVF method only estimates the orientation and it is defined between $-90^{\circ}$ and $90^{\circ}$ degrees, the direction of each vector can be obtained taking into account the location of the representative pixel of each branch in relation to the centre of the bifurcation. Considering the quadrant of the representative pixel, the direction of the vector can be obtained, providing a vector field which is defined between $0^{\circ}$ and $360^{\circ}$ degrees.

Finally, after the conversion of the orientation space, it is possible to calculate the angular difference between the vessels contained in the window and thus to obtain a measure of the bifurcation angle.

\section{RESULTS}

The performance of the proposed method has been evaluated on images belonging to DRIVE database (Staal et al., 2004). This database contains 40 retinal images of $565 \times 584$ pixels as well as their manual segmentation performed by experts. DRIVE is often used to evaluate segmentation algorithms designed specifically for the retinal vascular tree. In this paper, two methods for the computation of bifurcation angles have been compared: the proposed method based on the orientation vector field and the method described in (Morales et al., 2014).

Regarding the proposed method, it has been applied directly to the green component of the original RGB image. The directional openings were performed using an oriented linear structuring element of 7 pixels. This length comes from the trade-off between the curvature of the vessels and the angular resolution of the structuring element (it provides $\Delta \theta=$ $15^{\circ}$ and produces a filter bank with 12 branches). The low-pass filtering of the directional openings is performed using $\sigma=1$. The threshold $t h$ used to consider that a pixel belongs to a bifurcation or crossover is the
$25 \%$ of the maximum intensity value. In Figure 5, the angles measured on two images of DRIVE database can be observed. Only a region of interest of these images has been shown for better visualization.

The parameters of the orientation estimation have been chosen to deal with this particular resolution. However, the orientation estimation method can be improved by considering a multiscale approach as described in (Angulo et al., 2011). There, the estimation of the orientation properties is provided by directional openings by line segments of variable length, which produce directional signatures for various scales.

With respect to the algorithm presented in (Morales et al., 2014), the main difference is that it only can be applied to binary images. Moreover, once the yessels have been segmented with this approach, the retinal vascular tree must be labelled in order to perform the angle measurements. The vascular tree labelling is focused on obtaining the vascular skeleton, detecting its bifurcation points through hit-ormiss transformations and a tracking process. Then, the angle formed by the daughter branches of each bifurcation point is calculated. The branches are fitted by straight lines using least-squares in a circular window centred on these points. As it was mentioned in Section 1, the main drawback of the methods based on the vascular skeleton is its excessive dependence between the skeleton pixels and the measured angle. Figure 6 shows the measured angles provided by the method described in (Morales et al., 2014) on different expert hand-made segmentations.

Comparing the results shown in Figure 6, it can be appreciated that the measured angles can be quite different, reaching a maximum angle variation on the same image of around $7 \%$ in the Figure $6 a$ and around $6 \%$ in the Figure $6 \mathrm{~b}$. Note that the variation is due to the fact that the fitted line of the branches depends directly on the skeleton pixels considered, and the skeleton shape depends on the segmentation. Only the change in one pixel of the skeleton can modify the fitted line and therefore the calculated angle. The angles shown in the Figure $6 \mathrm{a}$ and $6 \mathrm{~b}$ correspond with the existing bifurcations within the red rectangles of the Figure 5a and 5b respectively. Since the method proposed in this paper is based on the grey image and therefore, it avoids the segmentation and skeletonization stages, the provided measurements are much more accurate, stable and faithful to reality.

\section{CONCLUSIONS}

This paper has addressed a novel method to measure bifurcation angles in retinal images. The method is 


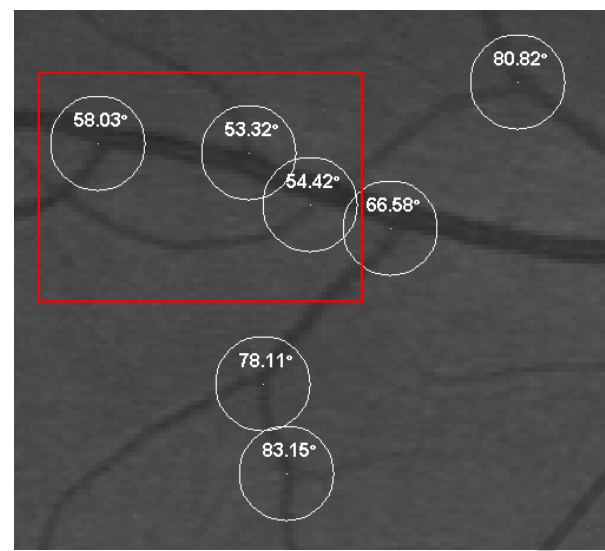

(a)

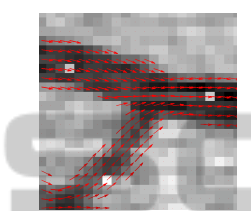

(c)

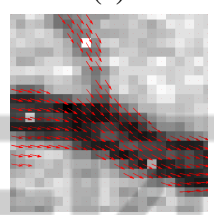

(d)

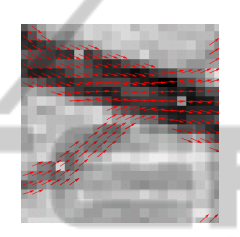

(e)

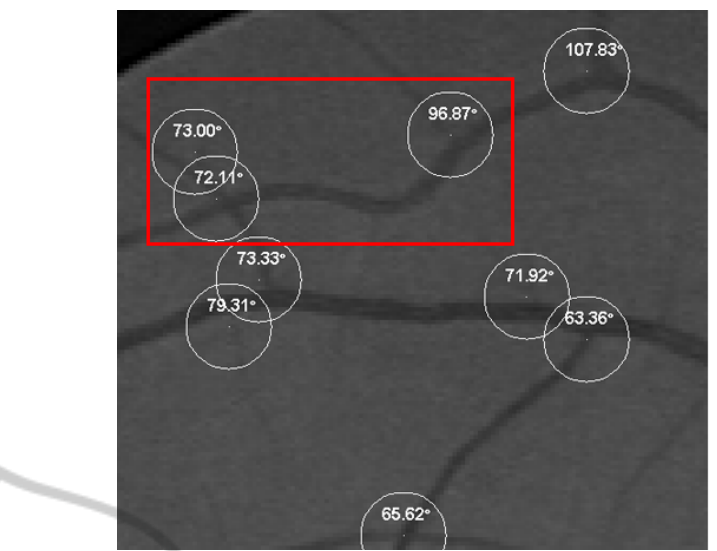

(b)

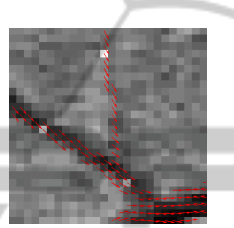

(f)

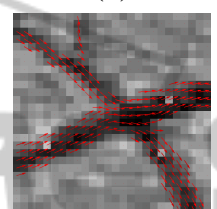

(g)

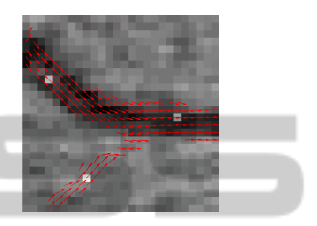

(h)

Figure 5: Angles of the bifurcations with the ASGVF orientation vector field on two images from DRIVE database. (a) A region of interest of the ' 19 test' image. (b) A region of interest of the ' 40 training' image. (c)-(e) Close up of selected bifurcations marked in red in (a). (f)-(h) Close up of selected bifurcations marked in red in (b). The bright pixels inside the vessels indicate at what points the value of the vector field has been taken.
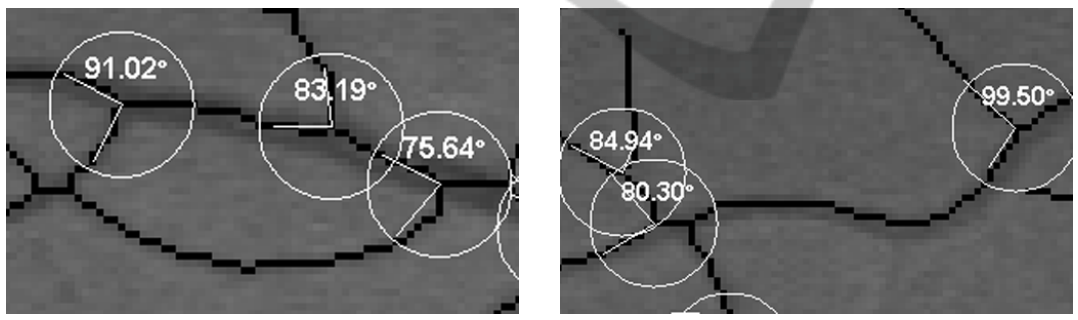

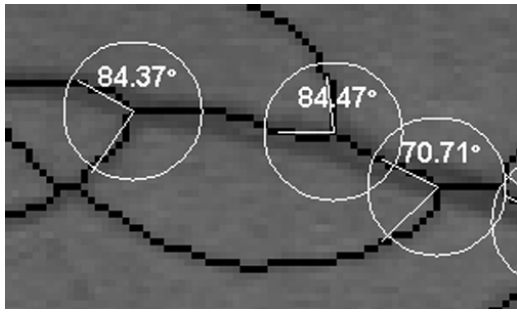

(a)

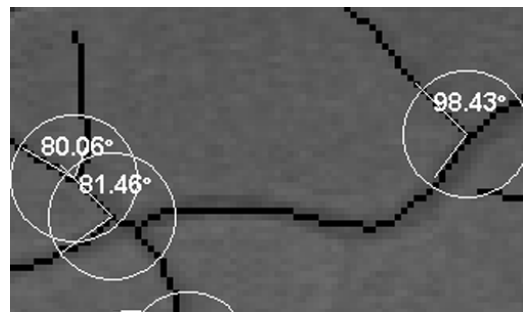

(b)

Figure 6: Bifurcation angles provided by the method described in (Morales et al., 2014) on the selected bifurcations of the Figure 5. This method is based on the skeletonization of a segmented (binary) image. (a) Skeletonization of two different expert segmentations of the ' 19 test' image. (b) Skeletonization of of two different expert segmentations of the ' 40 training' image. Slight differences in the skeleton cause large differences in the measured angles.

based on a multiple orientation vector field which is obtained from gray images. The coexistence at some locations of two or more orientations provides the interest points where a bifurcation can exist, but also high curvature vessels are wrongly provided. After a classification stage, the false positives are reduced and then the measurement is performed using the orientation estimation provided by the ASGVF of the gray image. Since the orientation method is based on the gradient of the gray image, no segmentation is required. The proposed method can measure accurately the bifurcation angles and it is more robust 
than other methods which use the skeleton of the segmented image. The measurements based on the skeleton depends highly on this process. The change of a single pixel of the skeleton can modify the line which fits the branch and therefore it involves variations in the angle measurement.

As future work, we will evaluate the performance of the proposed method on all retinal images from DRIVE (Staal et al., 2004) and STARE (Hoover et al., 2000) databases. Comparisons with other state-of-art methods will also be done.
Legaz-Aparicio, A. G., Verdú-Monedero, R., MoralesSánchez, J., Larrey-Ruiz, J., and Angulo, J. (2014). Detection of retinal vessel bifurcations by means of multiple orientation estimation based on regularized morphological openings. In XIII Mediterranean Conference on Medical and Biological Engineering and Computing 2013, volume 41 of IFMBE Proceedings, pages 317-320. Springer International Publishing.

MacGillivray, T., Perez-Rovira, A., Trucco, E., Chin, K., Giachetti, A., Lupascu, C., Tegolo, D., Wilson, P., Doney, R., Laude, A., and Dhillon, B. (2012). VAMPIRE: Vessel Assessment and Measurement Platform for Images of the Retina. CRC Press.

Martinez Perez, M., Highes, A., Stanton, A., Thorn, S., Chapman, N., Bharath, A., and Parker, K. (2002). Retinal vascular tree morphology: a semi-automatic quantification. IEEE Trans. on Biomedical Engineering, 49(8):912-917.

This work was supported by Ministerio de Economía y Competitividad of Spain, Project ACRIMA (TIN2013-46751-R).

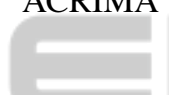

\section{REFERENCES}

Abramoff, M., Garvin, M., and Sonka, M. (2010). Retinal imaging and image analysis. Biomedical Engineering, IEEE Reviews in, 3:169-208.

Angulo, J., Verdú-Monedero, R., and Morales-Sánchez, J. (2011). Multiscale local multiple orientation estimation using Mathematical Morphology and B-spline interpolation. Proc. of 7 th Int. Symposium on Image and Signal Processing and Analysis (ISPA), pages 575 578.

Ardizzone, E., Pirrone, R., Gambino, O., and Radosta, S. (2008). Blood vessels and feature points detection on retinal images. Engineering in Medicine and Biology Society, 2008. EMBS 2008. 30th Annual International Conference of the IEEE, pages 2246-2249.

Azzopardi, G. and Petkov, N. (2011). Detection of retinal vascular bifurcations by trainable v4-like filters. CAIP'11 Proc. 14th Int. Conf. on Computer Analysis of Images and Patterns, 1:451-459.

Azzopardi, G. and Petkov, N. (2013). Automatic detection of vascular bifurcations in segmented retinal images using trainable cosfire filters. In Pattern Recognition Letters, volume 34, pages 922-933.

Chaudhuri, S., Chatterjee, S., Katz, N., Nelson, M., and Goldbaum, M. (1989). Detection of blood vessels in retinal images using two-dimensional matched filters. IEEE Trans. on Medical Imaging, 8:263-269.

Gao, X. W., Bharath, A., Stanton, A., Hughes, A., Chapman, N., and Thom, S. (2000). Quantification and characterisation of arteries in retinal images. Computer Methods and Programs in Biomedicine, 63(2):133 - 146.

Hoover, A., Kouznetsova, V., and Goldbaum, M. (2000). Locating blood vessels in retinal images by piecewise threshold probing of a matched filter response. IEEE Transactions on Medical Imaging, 19(3):203-210.
Martínez-Pérez, M. E., Hughes, A., Stanton, A., Thom, S., Bharath, A., and Parker, K. (1999). Scale-space analysis for the characterisation of retinal blood vessels. Medical Image Computing and Computer-Assisted Intervention, pages 90-97.

Morales, S., Naranjo, V., López-Mir, F., Navea, A., and niz, M. A. (2014). Computer-assisted system for hypertensive risk determination through fundus image processing. In Romero, L. M. R., editor, XIII Mediterranean Conference on Medical and Biological Engineering and Computing 2013, volume 41 of IFMBE Proceedings, pages 273-276. Springer.

Soille, P. and Talbot, H. (2001). Directional morphological filtering. IEEE Trans. on Pattern Analysis and Machine Intelligence, 23(11):1313-1329.

Staal, J., Abramoff, M., Niemeijer, M., Viergever, M., and van Ginneken, B. (2004). Ridge-based vessel segmentation in color images of the retina. Medical Imaging, IEEE Transactions on, 23(4):501-509.

Verdú-Monedero, R., Angulo, J., and Serra, J. (2011). Anisotropic morphological filters with spatiallyvariant structuring elements based on imagedependent gradient fields. IEEE Trans. Image Processing, 20(1):200-212.

Zana, F. and Klein, J.-C. (2001). Segmentation of vessellike patterns using mathematical morphology and curvature evaluation. IEEE Trans. on Image Processing, 10(7):1010-1019. 